\title{
Quels critères pour l'examen de spécialiste? (état 2011)
}

\author{
L'Institut suisse pour la formation médicale postgraduée et continue (ISFM) introduit \\ la clause éliminatoire pour les examens de spécialiste en chirurgie de la main et en \\ chirurgie orale et maxillo-faciale ainsi que pour l'examen de formation approfondie \\ en néphrologie pédiatrique.
}

\section{Christoph Hänggeli}

Directeur de l'Institut suisse pour la formation médicale postgraduée et continue (ISFM)
Le $1^{\text {er }}$ janvier 2011, l'ISFM a mis en vigueur la clause éliminatoire des examens de spécialiste et de formation approfondie pour trois nouvelles spécialités. Ainsi, la réussite de l'examen de spécialiste ou de la formation approfondie est désormais exigée pour tous les titres postgrades fédéraux et pour une partie des formations approfondies, sous réserve des dispositions transitoires applicables.

\section{Dispositions transitoires}

Font exception les candidats remplissant une des deux conditions suivantes:

- Les candidats ayant déjà participé (entièrement ou en partie) à un examen de spécialiste ou un examen de formation approfondie dans le domaine concerné avant l'entrée en vigueur du ca- ractère éliminatoire de l'examen ne devront pas passer d'autre examen.

- Les candidats terminant leur formation postgraduée dans les deux ans après l'entrée en vigueur du caractère éliminatoire de l'examen de spécialiste ne doivent attester, pour l'obtention du titre ou de la formation approfondie, que leur participation à l'examen. Attention: toutes les conditions doivent être remplies durant les deux ans qui suivent la date d'entrée en vigueur (y compris l'examen de spécialiste ou l'examen de formation approfondie complet, sauf si la société de discipline médicale refuse la participation à la $2^{\mathrm{e}}$ partie de l'examen en raison d'un résultat insuffisant à la $1^{\text {re }}$ partie)!
Correspondance: Christoph Hänggeli ISFM/FMH Elfenstrasse 18 CH-3000 Berne 15 Tél. 0313591111 Fax 0313591112 siwf@fmh.ch

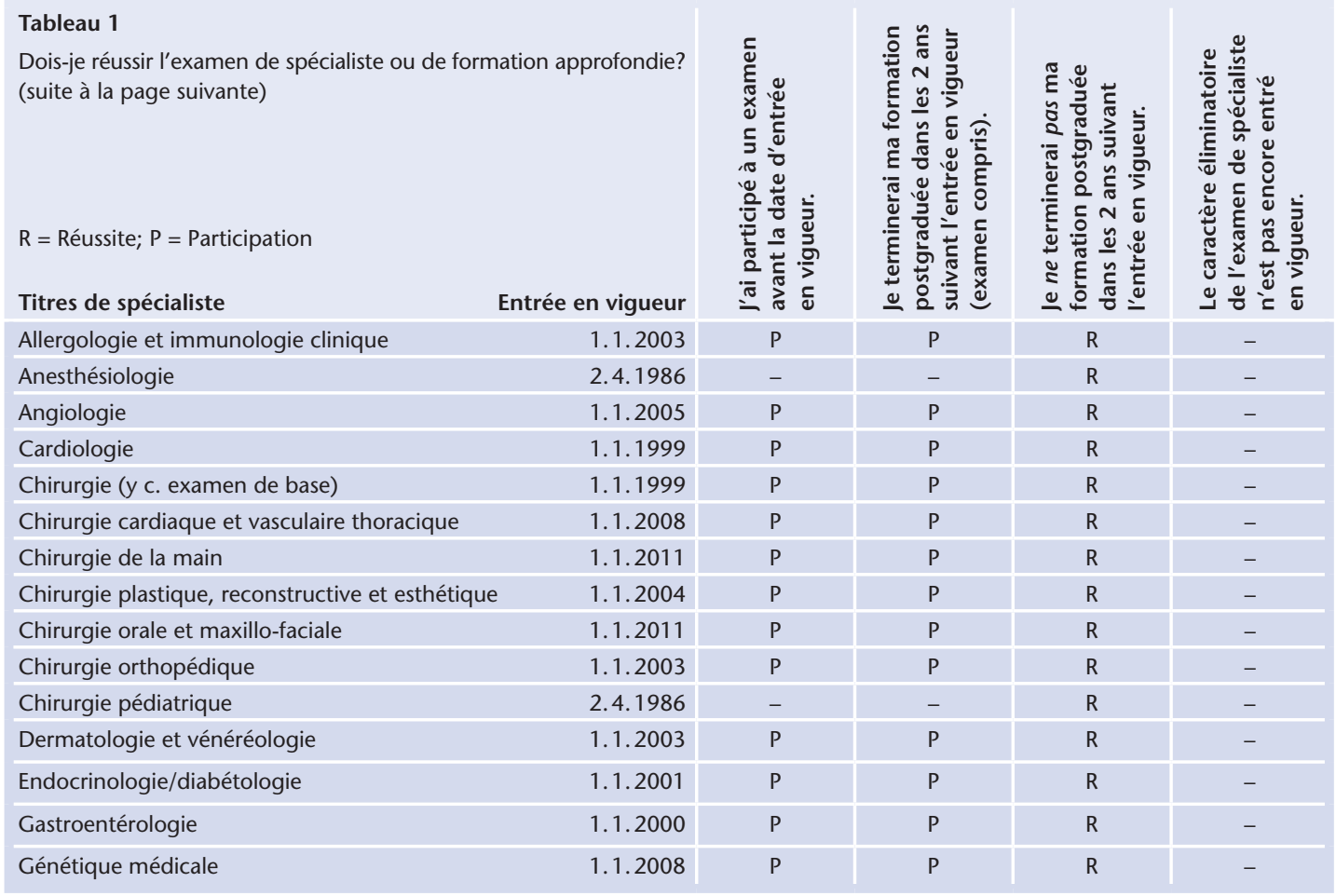




\section{Tableau}

Dois-je réussir l'examen de spécialiste ou de formation approfondie? (suite de la page précédente)

$\mathrm{R}=$ Réussite; $\mathrm{P}=$ Participation

Titres de spécialiste

Entrée en vigueur

Gynécologie et obstétrique

1.1 .1999

Hématologie

Infectiologie

Médecine du travail

1.1 .2001

1. 1.2003

Médecine générale

1. 1.2001

Médecine intensive

1. 1.2000

Médecine interne

1. 1.2001

Médecine légale

1. 1.1999

Médecine nucléaire

1.1.2001

Médecine pharmaceutique

2. 4.1986

Médecine physique et réadaptation

1. 1.2003

Médecine tropicale

1. 1.2003

Néphrologie

Neurochirurgie

Neurologie

1.1.2001

1.1.2009

2. 4.1986

Neuropathologie

1. 1.2003

Oncologie médicale

1.1.2012?

Ophtalmologie

1. 1.2001

ORL (sans la chirurgie maxillo-faciale)

Pathologie

Pharmacologie et toxicologie cliniques

1. 1.2002

1. 1.2001

1.1 .1999

Pédiatrie

Pneumologie

1.1 .2006

1. 1.2004

Prévention et santé publique

Psychiatrie et psychothérapie

Psychiatrie et psychothérapie

d'enfants et d'adolescents

Radiologie

Radio-oncologie / radiothérapie

1. 1.2003

1.1.2004

1.1. 2001

1.1.2002

2. 4.1986

Rhumatologie

2. 4.1986

1. 1.2001

Urologie

1.1.2002

Formations approfondies

Chirurgie générale et d'urgence

Chirurgie thoracique

Chirurgie vasculaire

Chirurgie viscérale

Endocrinologie-diabétologie pédiatrique

Gastroentérologie et hépatologie pédiatrique

Cardiologie pédiatrique

Gériatrie

Néonatologie

Néphrologie pédiatrique

Neuropédiatrie

Pathologie moléculaire

Urologie opératoire

Phoniatrie

Pneumologie pédiatrique

Radiologie pédiatrique

toutes les autres formations approfondies
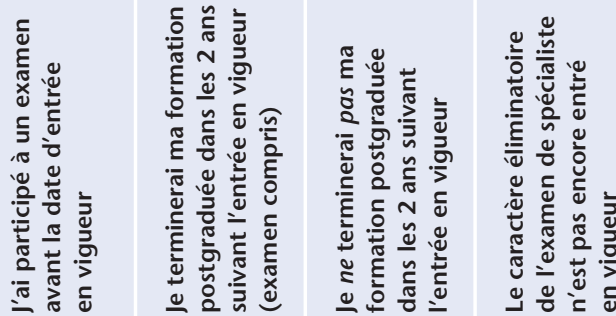

\section{$\mathrm{R}$}

$\mathrm{R}$

P

$P$

$P$

$P$

P

$P$

$P$

P

P

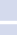

P

$P$

P

$P$

$P$

$P$

$P$

$P$

$\mathrm{P}$
$\mathrm{P}$

$x^{2}$

\begin{tabular}{l}
- \\
- \\
\hline$P$ \\
$P$
\end{tabular}

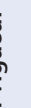

la formation postgraduée
(avec les dates actuelles
d'examen) se trouve sur le
site internet de la FMH à
I'adresse suivante: www.
siwf.ch. Les dates d'examen
sont aussi publiées dans le
Bulletin des médecins
suisses. Pour de plus amples
informations, veuillez vous
adresser à I'Institut suisse
pour la formation médicale
postgraduée et continue
(ISFM), case postale 170 , El-
fenstr. 18, 3000 Berne 15 ,
tél. 0313591111 , fax 031
3591112 , courriel: siwf@
fmh.ch

la formation postgraduée
(avec les dates actuelles
d'examen) se trouve sur le
site internet de la FMH à
I'adresse suivante: www.
siwf.ch. Les dates d'examen
sont aussi publiées dans le
Bulletin des médecins
suisses. Pour de plus amples
informations, veuillez vous
adresser à I'Institut suisse
pour la formation médicale
postgraduée et continue
(ISFM), case postale 170 , El-
fenstr. 18, 3000 Berne 15 ,
tél. 0313591111 , fax 031
3591112 , courriel: siwf@
fmh.ch

la formation postgraduée
(avec les dates actuelles
d'examen) se trouve sur le
site internet de la FMH à
I'adresse suivante: www.
siwf.ch. Les dates d'examen
sont aussi publiées dans le
Bulletin des médecins
suisses. Pour de plus amples
informations, veuillez vous
adresser à I'Institut suisse
pour la formation médicale
postgraduée et continue
(ISFM), case postale 170 , El-
fenstr. 18, 3000 Berne 15 ,
tél. 0313591111 , fax 031
3591112 , courriel: siwf@
fmh.ch

la formation postgraduée
(avec les dates actuelles
d'examen) se trouve sur le
site internet de la FMH à
I'adresse suivante: www.
siwf.ch. Les dates d'examen
sont aussi publiées dans le
Bulletin des médecins
suisses. Pour de plus amples
informations, veuillez vous
adresser à I'Institut suisse
pour la formation médicale
postgraduée et continue
(ISFM), case postale 170 , El-
fenstr. 18, 3000 Berne 15 ,
tél. 0313591111 , fax 031
3591112 , courriel: siwf@
fmh.ch la formation postgraduée
(avec les dates actuelles
d'examen) se trouve sur le
site internet de la FMH à
I'adresse suivante: www.
siwf.ch. Les dates d'examen
sont aussi publiées dans le
Bulletin des médecins
suisses. Pour de plus amples
informations, veuillez vous
adresser à I'Institut suisse
pour la formation médicale
postgraduée et continue
(ISFM), case postale 170, El-
fenstr. 18, 3000 Berne 15 ,
tél. 0313591111 , fax 031
3591112, courriel: siwf@
fmh.ch
1. 1.2009

1. 1.2007

1.1.2007

1. 1.2009

1.1.2005

1. 1.2010

1. 1.2001

1.1.2006

1.1.2003

1. 1.2011

1. 1.2003

1. 1.2010

1.1. 2009

1. 1.2007

1.1. 2009

1.1.2006

\begin{tabular}{|c|c|}
\hline P \\
\hline P \\
\hline$P$ \\
\hline$P$ \\
\hline$P$ \\
\hline$P$ \\
\hline$P$ \\
\hline$P$ \\
\hline$P$ \\
\hline$P$ \\
\hline$P$ \\
\hline$P$ \\
\hline$P$
\end{tabular}

